\title{
MiR-542-5p regulates the progression of diabetic retinopathy by targeting CARM1
}

\author{
Ning Guo, Ayinu Nulahou, Qian Bu, Meng Liu, Yan Wang, Yong Zhao, Lei Yang \\ and Yunxian Gao®
}

Department of Ophthalmology, Traditional Chinese Medical Hospital of Xinjiang Uygur Autonomous Region, Urumqi, Xinjiang Uygur Autonomous Region, 830000, China

\begin{abstract}
Diabetic retinopathy (DR), as the most frequent microvascular complication of diabetes mellitus (DM), causes vision loss and blindness in adults worldwide with increasing incidence. MicroRNAs (miRNAs) are involved in the regulation of DR. However, the role of miR-542-5p is still unknown. Here, we demonstrate that miR-542-5p is down-regulated in patients with DR and in high-glucose (HG)-treated retinal pigment epithelial cells. Moreover, miR-542-5p overexpression inhibits apoptosis in retinal pigment epithelial cells exposed to HG. The interaction between miR-542-5p and co-activator-associated arginine methyltransferase 1 (CARM1) is confirmed. MiR-542$5 p$ mimics decrease the CARM1 level and miR-542-5p inhibitor increases the CARM1 level. Additionally, CARM1 overexpression promotes the miR-542-5p-mediated apoptosis in HG-treated retinal pigment epithelial cells. In summary, the data suggest that miR-542-5p may suppress apoptosis in retinal pigment epithelial cells via targeting CARM1, which provides a new therapeutic target for the treatment of patients with DR.
\end{abstract}

Key words: Diabetic retinopathy, miR-542-5p, CARM1, apoptosis

Received: 31 March, 2020; revised: 28 May, 2020; accepted: 21 July, 2020; available on-line: 01 September, 2020

๑e-mail: FGHUG78ui@163.com

Abbreviations: CARM1, co-activator-associated arginine methyltransferase 1; DR, diabetic retinopathy; DM, diabetes mellitus; $E C L$, enhanced chemiluminescence; HG, high-glucose; miRNAs, microRNAs; NDR, non-diabetic retinopathy; PI, propidium iodide; qRT-PCR, quantitative real-time PCR; UTR, untranslated region

\section{INTRODUCTION}

Diabetes is considered the most common metabolic disease in the world with increasing mortality (Ravindran et al., 2015; Wild et al., 2004). Diabetic retinopathy (DR), as the most frequent microvascular complication of diabetes mellitus (DM), is characterized by hemorrhages, lipid exudates and neovascularization (Shao \& Yao, 2016; Barot et al., 2013). DR mainly causes vision loss and blindness in adults worldwide (Chong et al., 2017; Klein, 2007; Cheung et al., 2010). The incidence of DR has been increasing annually worldwide (Wang et al., 2012; Klein et al., 2017). Laser photocoagulation treatment shows no significant improvement in visual acuity in the long term (Liu et al., 2019). Therefore, it is necessary to further investigate more efficient therapeutic strategies to improve the treatment of patients with DR.

MicroRNAs (miRNAs) represent a group of highly conserved short (19-25 nucleotides) non-coding RNAs that bind to the 3'-untranslated region (UTR) of mRNAs to suppress gene expression in various cell processes ( $\mathrm{Li}$ \& Liu, 2011; Bartel, 2004; Hwang \& Mendell, 2007). Accumulating evidence has shown that miRNAs are involved in the regulation of DR (Hagiwara et al., 2013, Wu et al., 2014). For example, miR-590-3p has been found to elevate cell viability and repress pyroptosis in DR (Gu et al., 2019). Moreover, miR-203-3p represses oxygen-induced retinopathy-induced retinal angiogenesis and improves proliferative diabetic retinopathy (Han et al., 2020). A previous study also shows that miR-542$5 \mathrm{p}$ plays a suppressive role in neuroblastoma (Bray et al., 2011). Zhu and others (Zhu et al., 2020) found that miR-542-5p mediates osteosarcoma tumorigenesis and enhances proliferation in osteosarcoma. These findings indicate that miR-542-5p may take part in the development of various diseases. Interestingly, $\mathrm{Wu}$ and others (Wu et al., 2012) observed that miR-542-5p is abnormally expressed in DR. Therefore, we hypothesize that miR542-5p may play an important role in the development of DR.

Co-activator-associated arginine methyltransferase 1 (CARM1) is a coactivator of transcriptional activation and forms homodimers, which is necessary for catalytic activity (Chen et al. 1999; Higashimoto et al., 2007). Recently, CARM1 was found to be increased in patients with diabetes (Porta et al., 2019). Additionally, CARM1 is elevated and participates in the regulation of high-glucose (HG) treated human retinal pigment epithelial cells (Kim et al., 2014). These results suggest that CARM1 may be closely associated with the development of DR.

Here, we explore the role of miR-542-5p and CARM1 in HG-treated human retinal pigment epithelial cells. Our study suggests that miR-542-5p may be involved in the development of DR by targeting CARM1.

\section{MATERIALS AND METHODS}

Clinical samples. A total of 79 patients with DM (34 patients without DR and 45 patients with DR) and 40 healthy volunteers were from Traditional Chinese Medical Hospital of Xinjiang Uygur Autonomous Region. The blood samples from patients were collected and then stored at $-80^{\circ} \mathrm{C}$ until use. In this study, all patients signed informed consent. The experiments conform to the World Medical Association Declaration of Helsinki and were approved by the Ethics Committee of Traditional Chinese Medical Hospital of Xinjiang Uygur Autonomous Region.

Cell culture, treatment and transfection. The human retinal pigment epithelial line ARPE-19 was purchased from ATCC (Manassas, VA, USA), and cultured 
in DMEM containing $10 \%$ fetal bovine serum at $37^{\circ} \mathrm{C}$ under $5 \% \mathrm{CO}_{2}$. The cells were treated with $5 \mathrm{mM}$ glucose (Control) or $25 \mathrm{mM}$ glucose (HG) for $24 \mathrm{~h}$. Additionally, the cells were transfected with miR-542-5p mimics or negative control mimics (NC mimics) for $24 \mathrm{~h}$ and then exposed to HG for another $24 \mathrm{~h}$.

Quantitative real-time PCR (qRT-PCR). Total RNA was extracted using TRIzol reagent (Takara, Dalian, China). Then, cDNAs were synthesized through Taqman MicroRNA assays (Beyotime, Shanghai, China). The qRT-PCR was performed, and miR-542-5p and

\begin{tabular}{ll}
\hline Name & Sequences $\left(5^{\prime}-3^{\prime}\right)$ \\
\hline miR-542-5p & F: CTCCTCTCGGGGATCATCAT \\
& R: TATGGTTGTTCACGACTCCTTCAC \\
\hline CARM1 & F: TTGATGTTGGCTGTGGCTCTGG \\
& R: ATGGGCTCCGAGATGATGATGTCC \\
U6 & F: CTCGCTTCGGCAGCACATATACT \\
\hline GAPDH & F: GCACCGTCAAGGCTGAGAAC \\
& R: GGATCTCGCTCCTGGAAGATG \\
\hline
\end{tabular}

CARM1 levels were determined via SYBR Green ( $\mathrm{Ta}$ kara, Dalian, China). The relative expression of miR-542$5 \mathrm{p}$ and CARM1 was calculated via the $2^{-\Delta \Delta \mathrm{CT}}$ method. The primers were as follows:

Western blot analysis. Protein was extracted via RIPA lysis buffer (Beyotime, Shanghai, China). Then, the protein concentration was quantified using BCA Kit (Takara, Dalian, China). Proteins were separated by SDS-PAGE and wet-transferred onto PVDF membrane (Beyotime, Shanghai, China). After blocking in 5\% nonfat milk for $1 \mathrm{~h}$, the membrane was incubated with primary antibodies such as CARM1 (1:5000), Cleaved caspase 3 (1:500), caspase 3 (1:1000), Bcl-2 (1:1000) and $\beta$-actin (1:5000) (all from Abcam, Shanghai, China), at $4 \mathrm{C}$ overnight. $\beta$-actin was used as the internal control. Subsequently, the membrane was incubated with the secondary antibody (1:3000, Beyotime, Shanghai, China) for $1 \mathrm{~h}$ at $37 \mathrm{C}$. The bands were visualized via enhanced chemiluminescence (ECL) reaction solution (Takara, Dalian, China).

Cell apoptosis analysis. Cells $\left(3 \times 10^{4} / \mathrm{ml}\right)$ were collected and resuspended in $1 \times$ Annexin binding buffer $(500 \mu \mathrm{l})$, and stained with $5 \mu \mathrm{l}$ Annexin-V-FITC and $5 \mu l$ propidium iodide (PI) for $25 \mathrm{~min}$. Then, cell apoptosis was measured via flow cytometry (BD, Japan).

Luciferase reporter assay. TargetScan software (http://www.targetscan.org/vert_72/) predicted that miR-542-5p could target CARM1. The wild-type 3'-UTR sequence of CARM1 containing the miR-542-5p binding site, and the mutant 3'-UTR sequence of CARM1 lacking the miR-542-5p binding site were inserted into the pmirGLO reporter vector (pmirGLO-CARM1-WT and pmirGLO-CARM1-MUT). The ARPE-19 cells were cotransfected with pmirGLO-Report constructs and miR542-5p mimics or NC mimics using Lipofectamine 2000 (Beyotime, Shanghai, China) for $48 \mathrm{~h}$. The firefly and Renilla luciferase activities were examined through the Dual-Luciferase Reporter Assay System. The Renilla/ firefly luciferase ratio was analyzed.

Statistical analysis. Data were presented as mean \pm S.D. GraphPad Prism 6.0 was used to carry out statistical analysis. Statistical significance was assessed using Student's $t$-test and one-way ANOVA followed by the Bonferroni test. The correlation between the two groups was confirmed through Pearson's correlation analysis. $P<0.05$ was considered statistically significant.

\section{RESULTS}

\section{The effect of HG treatment on apoptosis of retinal pigment epithelial cells}

We first measured the level of miR-542-5p in patients with DR and HG-treated retinal pigment epithelial cells. qRT-PCR showed that the miR-542-5p level is lower in diabetes mellitus (DM)+non-diabetic retinopathy (NDR) group than in the Healthy group, and miR$542-5 p$ is reduced in patients with DM and DR compared with patients with DM. Moreover, the miR-542-5p in DM+NDR group is reduced compared with that in the Healthy group (Fig. 1A). The results from qPCR assay also show that miR-542-5p is reduced in HG-treated ARPE-19 cells compared with control (Fig. 1B). Cell
A

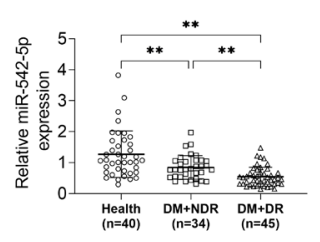

B

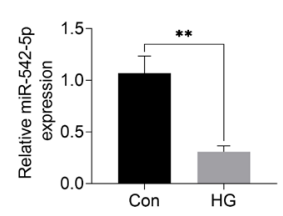

C
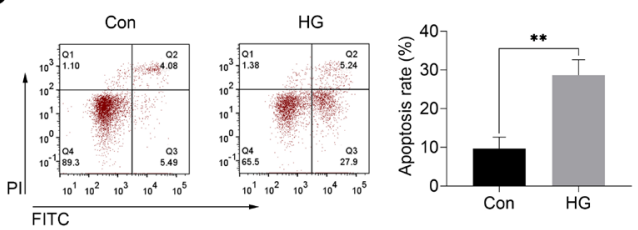

$\mathrm{D}$

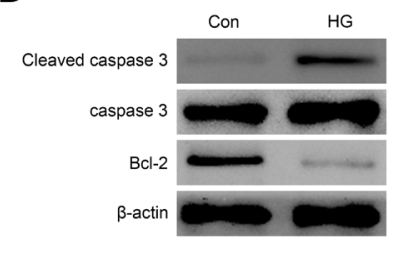

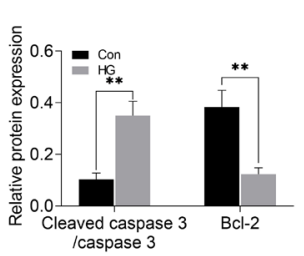

Figure 1. The effect of HG treatment on apoptosis of retinal pigment epithelial cells.

(A, B) MiR-542-5p mRNA level was measured using qRT-PCR. (C) Cell apoptosis analysis was performed via Annexin-V-FITC/PI staining. (D) Western blot analysis verified Cleaved caspase 3 and $\mathrm{BCl}-2$ protein levels. $\mathrm{n}=3$. ${ }^{*} P<0.01$. 
A

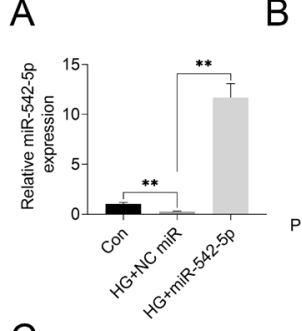

C

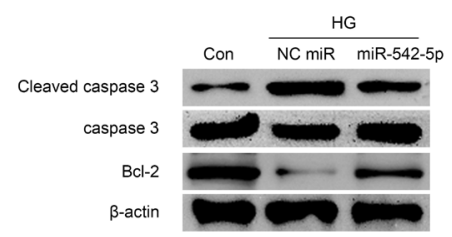

B
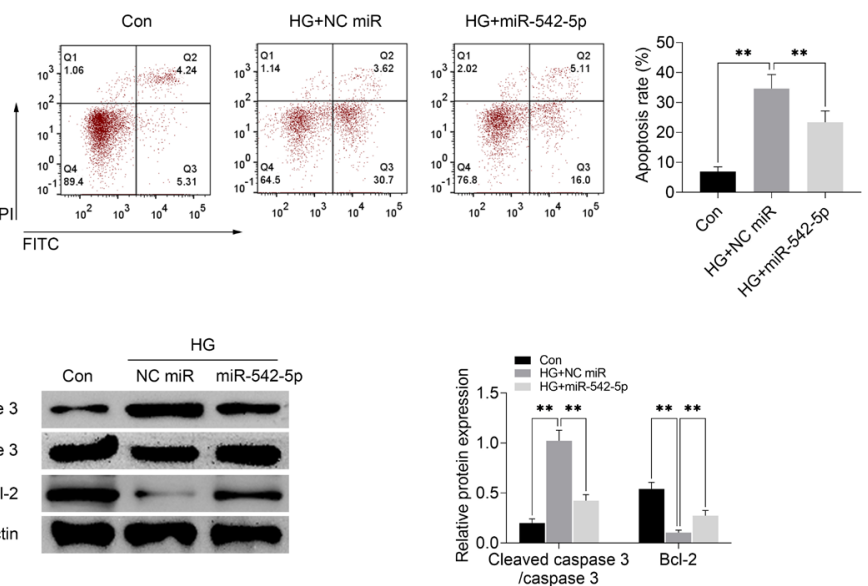

Figure 2. MiR-542-5p inhibits apoptosis in HG-treated retinal pigment epithelial cells.

(A) The qRT-PCR was used to measure miR-542-5p mRNA level. (B) Annexin-V-FITC/PI staining was carried out to examine cell apoptosis. (C) Cleaved caspase 3 and $\mathrm{BCl}-2$ protein levels were measured by western blot analysis. $\mathrm{n}=3$. ${ }^{* *} P<0.01$.

apoptosis analysis reveals that apoptosis is enhanced in HG-treated ARPE-19 cells (Fig. 1C). Western blot analysis indicates that HG treatment causes the elevation of Cleaved caspase 3 protein level and the reduction of $\mathrm{Bcl}-$ 2 protein level in ARPE-19 cells (Fig. 1D). These findings imply that miR-542-5p might be related with apoptosis in HG-treated retinal pigment epithelial cells.

\section{MiR-542-5p inhibits apoptosis in HG-treated retinal pigment epithelial cells}

To explore the effect of miR-542-5p on the apoptosis of HG-treated retinal pigment epithelial cells, we overexpressed miR-542-5p in HG-treated ARPE-19 cells. The qRT-PCR assay demonstrates that HG treatment results in the decrease of miR-542-5p, whereas miR- 542-5p mimics significantly elevate miR-542-5p level in HG-treated ARPE-19 cells (Fig. 2A). Annexin-V-FITC/ PI staining shows that cell apoptosis is enhanced after HG treatment, whereas miR-542-5p mimics repress cell apoptosis in ARPE-19 cells treated with HG (Fig. 2B). Consistently, western blot analysis reveals that miR-542$5 \mathrm{p}$ mimics inhibit the $\mathrm{HG}$-induced increase of Cleaved caspase 3 and $\mathrm{HG}$-induced reduction of $\mathrm{Bcl}-2$ protein level in ARPE-19 cells (Fig. 2C). These data indicate that miR-542-5p suppresses apoptosis of HG-treated retinal pigment epithelial cells.

\section{CARM1 is verified as a target of miR-542-5p}

We predict that CARM1 is a target of miR-542-5p using Targetscan analysis (Fig. 3A). The interaction be-
A

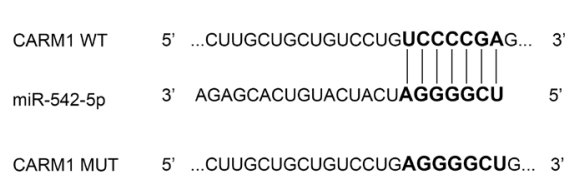

C

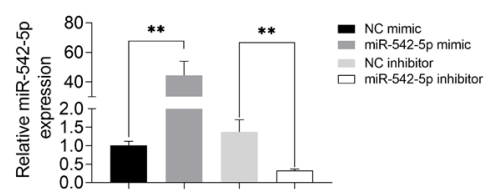

$E$

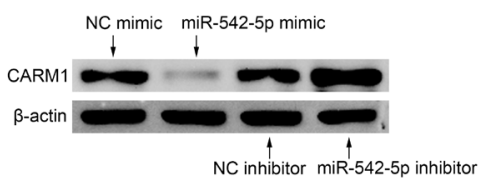

$\mathrm{B}$

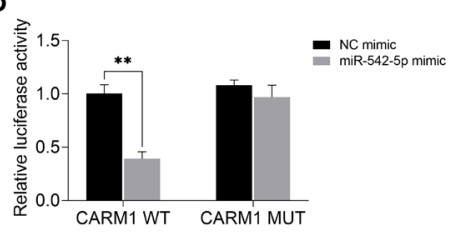

$\mathrm{D}$
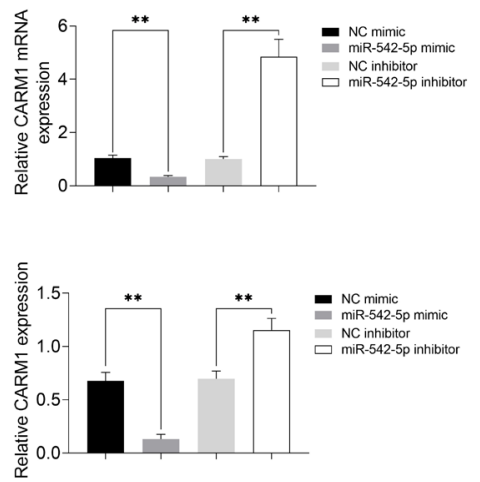

Figure 3. CARM1 is verified as a target of miR-542-5p.

(A) Targetscan analysis predicted that CARM1 was a target of miR-542-5p. (B) The interaction between miR-542-5p and CARM1 was confirmed through luciferase reporter assay. (C) MiR-542-5p mRNA level was measured via qRT-PCR. (D, E) CARM1 mRNA level and protein level were measured using qRT-PCR and western blot analysis, respectively. $n=3$. ${ }^{* *} P<0.01$. 
A
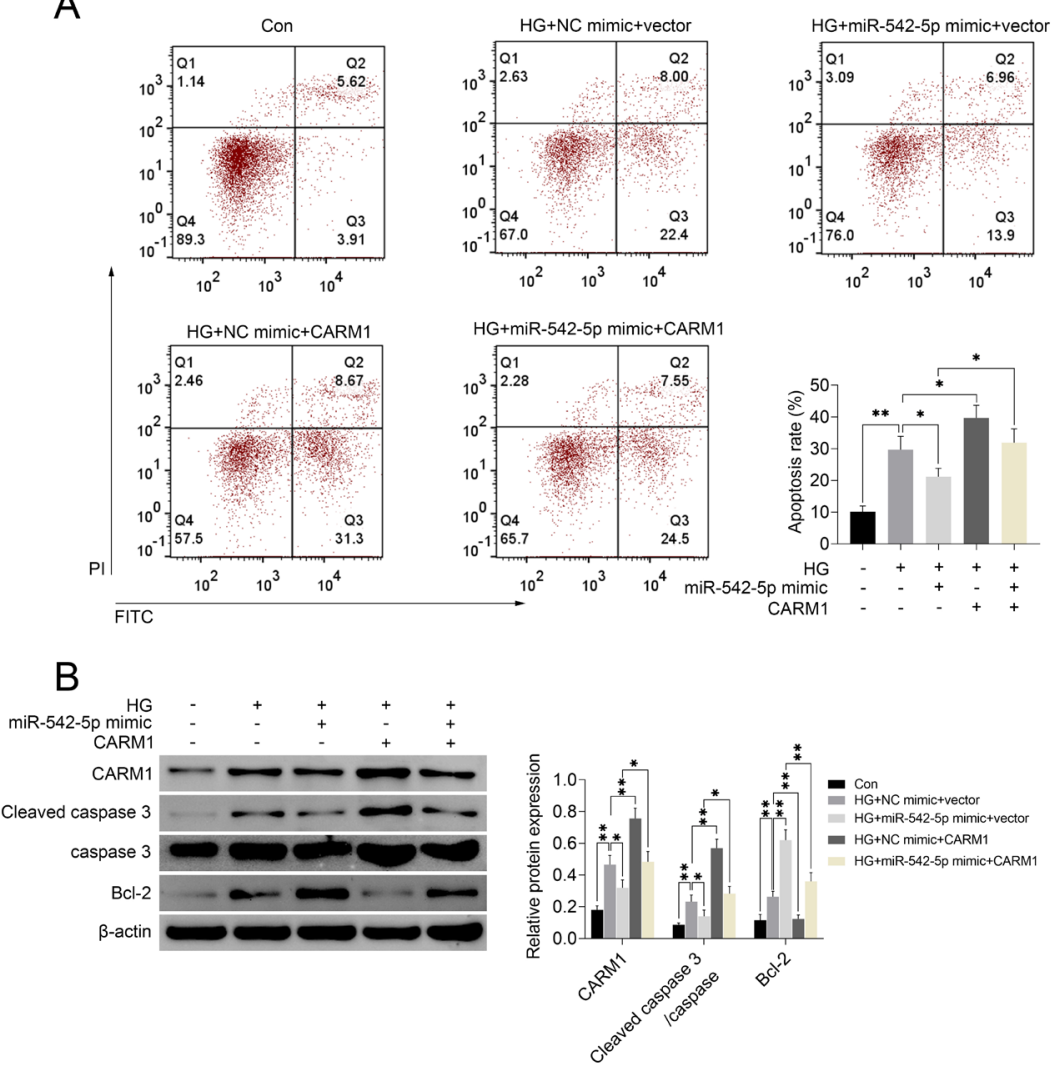

Figure 4. CARM1 overexpression attenuate the miR-542-5p-mediated apoptosis in HG-treated retinal pigment epithelial cells.

(A) Cell apoptosis was examined through Annexin-V-FITC/PI staining. (B) CARM1, Cleaved caspase 3 and Bcl-2 protein levels were determined using western blot analysis. $n=3 .{ }^{*} P<0.05$. ${ }^{* *} P<0.01$.

tween miR-542-5p and CARM1 is identified using the luciferase reporter assay. The results show that the luciferase activity is decreased in ARPE-19 cells co-transfected with miR-542-5p mimics and CARM1 WT 3'-UTR luciferase reporter vector, whereas there is no effect on the luciferase activity of ARPE-19 cells co-transfected with miR-542-5p mimics and CARM1 MUT3'-UTR luciferase reporter vector (Fig. 3B). qRT-PCR results demonstrate that miR-542-5p mimics increase the miR-542$5 p$ level, whereas miR-542-5p inhibitor decreases the miR-542-5p level (Fig. 3C). Western blot analysis and qRT-PCR show that miR-542-5p mimics repress the CARM1 level and miR-542-5p inhibitor increases the CARM1 level (Fig. 3D, E). These results indicate that CARM1 is a target of miR-542-5p and is negatively regulated by miR-542-5p.

\section{CARM1 overexpression attenuates the miR-542-5p- mediated apoptosis in HG-treated retinal pigment epithelial cells}

To test whether CARM1 can participate in the regulation of apoptosis induced by miR-542-5p in HGtreated retinal pigment epithelial cells, we performed Annexin-V-FITC/PI staining. The results show that miR-542-5p mimics inhibit apoptosis, and CARM1 overexpression promotes apoptosis in HG treated ARPE-19 cells. Moreover, CARM1 overexpression enhances miR542-5p-mediated apoptosis in HG-treated ARPE-19 cells (Fig. 4A). Additionally, western blot analysis proves that miR-542-5p mimics lower the CARM1 and Cleaved caspase 3 protein levels, and increase the $\mathrm{Bcl}-2$ protein level, whereas CARM1 overexpression has. the opposite effect on these protein levels. Further, CARM1 overexpression rescues these protein levels induced by miR542-5p mimics in HG-treated ARPE-19 cells (Fig. 4B). The data imply that CARM1 overexpression elevates the miR-542-5p-mediated apoptosis in HG-treated retinal pigment epithelial cells.

\section{DISCUSSION}

Here, we demonstrate that miR-542-5p is reduced in patients with DR and in HG-treated retinal pigment epithelial cells. Functional analysis indicated that miR-542$5 p$ represses apoptosis in retinal pigment epithelial cells in the presence of HG. Moreover, the interaction between miR-542-5p and CARM1 was identified. Further, CARM1 overexpression promotes miR-542-5p-mediated apoptosis in retinal pigment epithelial cells exposed to HG. The data imply that miR-542-5p may function in the apoptosis of retinal pigment epithelial cells, which contributes to the treatment of patients with DR.

Increasing evidence has revealed that abnormal expression of miRNAs has a close association with DR. Some miRNAs are upregulated. For example, miR-218 expression is increased, and it represses proliferation as well as stimulates apoptosis in human retinal pigment epithelium cells (Yao et al., 2019). MiR-29b-3p is elevated in patients with DR, and enhances the ratio of $\mathrm{Bax} / \mathrm{Bcl}-2$ in human retinal microvascular endothelial cells (Zeng et al., 2019). Conversely, some miRNAs have the opposite role. For instance, miR-7 has been demonstrated to inhibit apop- 
A

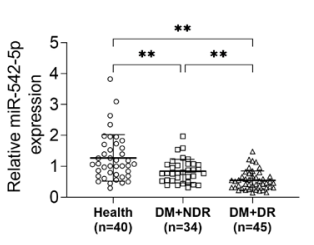

B

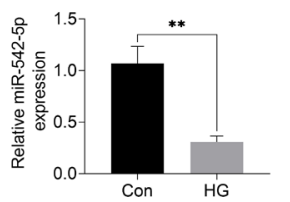

C
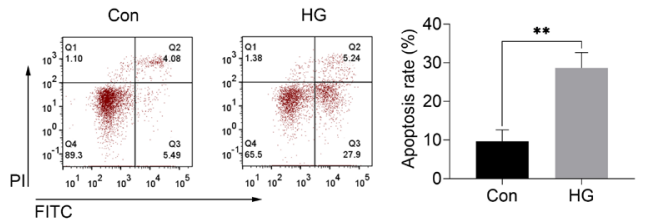

D
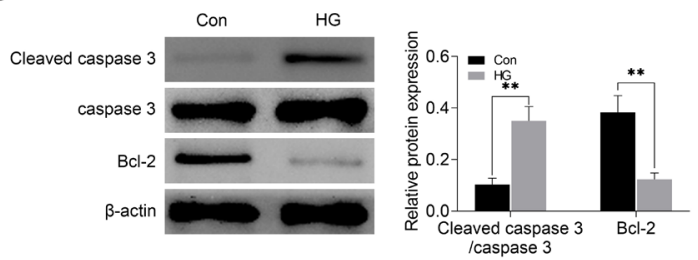

Figure 5. A schematic representation of the observed relationships between miR-542-5p and CARM1

tosis and alleviate $\mathrm{HG}$-induced damage in retinal pigment epithelial cells (Yang et al., 2019). MiR-142-5p is decreased in retinal tissues of DR rats and HG-treated human retinal endothelial cells, and improves pathological effects in retinal tissues (Liu et al., 2020). Like the later studies, our research verifies that miR-542-5p is suppressed in patients with DR and in HG exposed ARPE-19 cells. Moreover, miR-542-5p represses HGinduced apoptosis in ARPE-19 cells, accompanied by the reduction of the Cleaved caspase 3 protein level and elevation of the Bcl-2 protein level. These findings imply that miR-542-5p may be closely connected with the development of DR.

Previously, miRNAs were found to regulate the development of DR through targeting the 3'-UTR of mRNAs. For example, $\mathrm{Fu}$ and $\mathrm{Ou}(\mathrm{Fu} \& \mathrm{Ou}, 2020)$ found that miR-152 is reduced and represses HG-induced angiogenesis in human retinal endothelial cells through targeting LIN28B. Chen and others (Chen et al., 2019 discovered that miR-126 is decreased, and stimulates proliferation as well as suppresses apoptosis in HG-induced human retinal endothelial cells via targeting interleukin-17A). Interestingly, our study shows that miR-542-5p could target CARM1. Additionally, miR-542-5p down-regulates the CARM1 expression level. More importantly, accumulating evidence has shown that the function of miR-542-5p may vary in different diseases. For example, miR-542$5 \mathrm{p}$ has been found to alleviate fibroblast activation, and suppress proliferation and migration through targeting integrin $\alpha 6$ (Yuan et al., 2018). Conversely, Cheng and others (Cheng et al., 2015) have reported that miR-542$5 \mathrm{p}$ stimulates proliferation in osteosarcoma through targeting HUWE1. These findings suggest that miR-542-5p may regulate development of some diseases via targeting the target gene. Further analysis in this study proves that CARM1 overexpression increases the miR-542-5pmediated apoptosis in retinal pigment epithelial cells treated with HG. Notably, CARM1 was reported to be enhanced in HG-treated retinal pigment epithelial cells, and to promote apoptosis in retinal pigment epithelial cells (Kim et al., 2014). Moreover, Porta and others (Porta et al., 2019) demonstrated that the CARM1 expression level is high in type 2 diabetes. Our study is consistent with previous research. The data suggest that miR-542$5 p$ may take part in the inhibition of apoptosis in retinal pigment epithelial cells through targeting CARM1.
However, other miRNAs may also participate in regulating the progression of DR by targeting CARM1. Moreover, the clinical application of miR-542-5p was not evaluated, and the effect of miR-542-5p on apoptosis in patients with DR by targeting CARM1 was not explored in our study. Therefore, to further investigate the mechanism underlying miR-542-5p in patients with DR, more experiments will be carried out in the future.

In summary, the current study shows that miR-542-5p is down-regulated and CARM1 is up-regulated in HGtreated retinal pigment epithelial cells and in patients with DR. MiR-542-5p participates in the progression of DR via targeting CARM1 (Fig. 5), which could be useful for the treatment of patients with DR.

\section{Acknowledgements}

Not applicable.

\section{Funding}

None.

\section{Competing interests}

The authors state that there are no conflicts of interest to disclose.

\section{Ethics approval}

The experiments conformed to the World Medical Association Declaration of Helsinki and were approved by the Ethics Committee of Traditional Chinese Medical Hospital of Xinjiang Uygur Autonomous Region.

\section{Consent to participate}

All the patients signed written informed consent.

\section{Availability of data and materials}

All data generated or analyzed during this study are included in this published article.

\section{Authors' contributions}

Yunxian Gao and Ning Guo conceived and designed the experiments, Ayinu Nulahou, Meng Liu and Yan Wang analyzed and interpreted the results of the experi- 
ments, Qian Bu, Yong Zhao and Lei Yang performed the experiments

\section{REFERENCES}

Barot M, Gokulgandhi MR, Patel S, Mitra AK (2013) Microvascular complications and diabetic retinopathy: recent advances and future implications. Future Med Chem 5: 301-314. https://doi.org/10.4155/ fmc.12.206

Bartel DP (2004) MicroRNAs: genomics, biogenesis, mechanism, and function. Cell 116: 281-297. https://doi.org/10.1016/s00928674(04)00045-5.

Bray I, Tivnan A, Bryan K, Foley NH, Watters KM, Tracey L, Davidoff AM, Stallings RL (2011) MicroRNA-542-5p as a novel tumor suppressor in neuroblastoma. Cancer Lett 303: 56-64. https://doi. org/10.1016/j.canlet.2011.01.016.

Chen D, Ma H, Hong H, Koh SS, Huang SM, Schurter BT, Aswad DW, Stallcup MR (1999) Regulation of transcription by a protein methyltransferase. Science 284: 2174-2177. https://doi.org/10.1126/ science.284.5423.2174.

Chen X, Yu X, Li X, Li L, Li F, Guo T, Guan C, Miao L, Cao G (2019) MiR-126 targets IL-17A to enhance proliferation and inhibit apoptosis in high-glucose-induced human retinal endothelial cells. Biochem Cell Biol 98: 277-283. https://doi.org/10.1139/bcb-20190174

Cheng DD, Yu T, Hu T, Yao M, Fan CY, Yang QC (2015) MiR-542$5 \mathrm{p}$ is a negative prognostic factor and promotes osteosarcoma tumorigenesis by targeting HUWE1. Oncotarget 6: 42761-42772. https:// doi.org/10.18632/oncotarget.6199.

Cheung N, Mitchell P, Wong TY (2010) Diabetic retinopathy. Lancet 376: 124-136. https://doi.org/10.1016/S0140-6736(09)62124-3.

Chong YH, Fan Q, Tham YC, Gan A, Tan SP, Tan G, Wang JJ, Mitchell P, Wong TY, Cheng CY (2017) Type 2 diabetes genetic variants and risk of diabetic retinopathy. Ophthalmology 124: 336-342. https://doi.org/10.1016/j.ophtha.2016.11.016.

Fu X, Ou B (2020) miR-152/LIN28B axis modulates high-glucoseinduced angiogenesis in human retinal endothelial cells via VEGF signaling. J Cell Biochem 121: 954-962. https://doi.org/10.1002/ jcb. 28978 .

Gu C, Draga D, Zhou C, Su T, Zou C, Gu Q, Lahm T, Zheng Z, Qiu Q (2019) miR-590-3p Inhibits pyroptosis in diabetic retinopathy by targeting NLRP1 and inactivating the NOX4 signaling pathway. Invest Ophthalmol Vis Sci 60: 4215-4223. https://doi.org/10.1167/ iovs.19-27825.

Hagiwara S, McClelland A, Kantharidis P (2013) MicroRNA in diabetic nephropathy: renin angiotensin, aGE/RAGE, and oxidative stress pathway. J Diabetes Res 2013: 173783. https://doi. org $/ 10.1155 / 2013 / 173783$.

Han N, Xu H, Yu N, Wu Y, Yu L (2020) MiR-203a-3p inhibits retinal angiogenesis and alleviates proliferative diabetic retinopathy in oxygen-induced retinopathy (OIR) rat model via targeting VEGFA and HIF-1alpha. Clin Exp Pharmacol Physiol 47: 85-94. https://doi. org/10.1111/1440-1681.13163.

Higashimoto K, Kuhn P, Desai D, Cheng X, Xu W (2007) Phosphorylation-mediated inactivation of coactivator-associated arginine methyltransferase 1. Proc Natl Acad Sci U S A 104: 12318-12323. https://doi.org/10.1073/pnas.0610792104.

Hwang HW, Mendell JT (2007) MicroRNAs in cell proliferation, cell death, and tumorigenesis. Br J Cancer 96 (Suppl): R40-R44.

Kim DI, Park MJ, Lim SK, Choi JH, Kim JC, Han HJ, Kundu TK, Park JI, Yoon KC, Park SW, Park JS, Heo YR, Park SH (2014) High-glucose-induced CARM1 expression regulates apoptosis of human retinal pigment epithelial cells via histone 3 arginine 17 dimethylation: role in diabetic retinopathy. Arch Biochem Biophys 560: 36-43. https://doi.org/10.1016/j.abb.2014.07.021.
Klein BEK, Horak KL, Maynard JD, Lee KE, Klein R (2017) Association of skin intrinsic fluorescence with retinal microvascular complications of long term type 1 diabetes in the wisconsin epidemiologic study of diabetic retinopathy. Ophthalmic Epidemiol 24: 211-216. https://doi.org/10.1080/09286586.2016.1269934.

Klein BE (2007) Overview of epidemiologic studies of diabetic retinopathy. Ophthalmic Epidemiol 14: 179-183. https://doi. org/10.1080/09286580701396720.

Li L, Liu Y (2011) Diverse small non-coding RNAs in RNA interference pathways. Methods Mol Biol 764: 169-182. https://doi. org/10.1007/978-1-61779-188-8_11.

Liu P, Jia SB, Shi JM, Li WJ, Tang LS, Zhu XH, Tong P (2019) LncRNA-MALAT1 promotes neovascularization in diabetic retinopathy through regulating miR-125b/VE-cadherin axis. Biosci Rep 39: BSR20181469. https://doi.org/10.1042/BSR20181469.

Liu X, Li J, Li X (2020) miR-142-5p regulates the progression of diabetic retinopathy by targeting IGF1. Int J Immunopathol Pharmacol 34: 2058738420909041 . https://doi.org/10.1177/2058738420909041.

Porta M, Amione C, Barutta F, Fornengo P, Merlo S, Gruden G, Albano L, Ciccarelli M, Ungaro P, Durazzo M, Beguinot F, Berchialla P, Cavallo F, Trento M (2019) The co-activator-associated arginine methyltransferase 1 (CARM1) gene is overexpressed in type 2 diabetes. Endocrine 63: 284-292. https://doi.org/10.1007/s12020-0181740-z.

Ravindran R, Gopinathan DM, Sukumaran S (2015) Estimation of salivary glucose and glycogen content in exfoliated buccal mucosal cells of patients with type II diabetes mellitus. J Clin Diagn Res 9: ZC89ZC93. https://doi.org/10.7860/JCDR/2015/11633.5971.

Shao J, Yao Y (2016) Transthyretin represses neovascularization in diabetic retinopathy. Mol Vis 22: 1188-1197.

Wang YX, Xu L, Sun XY, Zou Y, Zhang HT, Jonas JB (2012) Five year incidence of visual field loss in adult Chinese. The Beijing Eye Study. PLoS One 7: e37232. https://doi.org/10.1371/journal. pone.0037232.

Wild S, Roglic G, Green A, Sicree R, King H (2004) Global prevalence of diabetes: estimates for the year 2000 and projections for 2030. Diabetes Care 27: 1047-1053. https://doi.org/10.2337/diacare.27.5.1047.

Wu H, Kong L, Zhou S, Cui W, Xu F, Luo M, Li X, Tan Y, Miao L (2014) The role of microRNAs in diabetic nephropathy. J Diabetes Res 2014: 920134. https://doi.org/10.1155/2014/920134.

Wu JH, Gao Y, Ren AJ, Zhao SH, Zhong M, Peng YJ, Shen W, Jing M, Liu L (2012) Altered microRNA expression profiles in retinas with diabetic retinopathy. Ophthalmic Res 47: 195-201. https://doi. org/10.1159/000331992.

Yang Z, Hu H, Zou Y, Luo W, Xie L, You Z (2019) miR-7 reduces high glucose induced-damage via HoxB3 and PI3K/AKT/mTOR signaling pathways in retinal pigment epithelial cells. Curr Mol Med 20: 372-378. https://doi.org/10.2174/156652401966619102315113 7.

Yao R, Yao X, Liu R, Peng J, Tian T (2019) Glucose-induced microRNA-218 suppresses the proliferation and promotes the apoptosis of human retinal pigment epithelium cells by targeting RUNX2. Biosci Rep 39: BSR20192580. https://doi.org/10.1042/BSR20192580.

Yuan J, Li P, Pan H, Li Y, Xu Q, Xu T, Ji X, Liu Y, Yao W, Han L, Ni C (2018) miR-542-5p Attenuates fibroblast activation by targeting integrin alpha6 in silica-induced pulmonary fibrosis. Int J Mol 19: 3717. https://doi.org/10.3390/ijms19123717.

Zeng Y, Cui Z, Liu J, Chen J, Tang S (2019) MicroRNA-29b-3p Promotes human retinal microvascular endothelial cell apoptosis via blocking SIRT1 in diabetic retinopathy. Front Physiol 10: 1621. https://doi.org/10.3389/fphys.2019.01621.

Zhu T, Fan D, Ye K, Liu B, Cui Z, Liu Z, Tian Y (2020) Role of miRNA-542-5p in the tumorigenesis of osteosarcoma. FEBS Open Bio 10: 627-636. https://doi.org/10.1002/2211-5463.12824. 